\title{
Applying a Self-Made Automobile Heterodyne Interferometer to Measure the Parameters of Twisted Nematic Liquid Crystal
}

\author{
Shinn-Fwu Wang, Pei-Cheng Ke, Wesley Lai, Hao Ma, Yi-Zhan Su, and Jyh-Shyan Chiu \\ Department of Electronic Engineering, Chien Hsin University of Science and Technology, No. 229 Chien-Hsin Road, \\ Taoyuan, Jhongli City 320, Taiwan \\ Correspondence should be addressed to Shinn-Fwu Wang, sfwangking@gmail.com
}

Received 13 July 2012; Revised 28 September 2012; Accepted 5 October 2012

Academic Editor: Ashish K. Prajapati

Copyright (๑) 2012 Shinn-Fwu Wang et al. This is an open access article distributed under the Creative Commons Attribution License, which permits unrestricted use, distribution, and reproduction in any medium, provided the original work is properly cited.

A self-made automobile heterodyne interferometer with a closed control loop is presented. In addition, we will apply the self-made automobile heterodyne interferometer to measure the parameters of optically compensated twisted nematic liquid crystal (TN LC). It is worthy to be mentioned that the self-made automobile heterodyne interferometer has a compact size because all the optical components are installed in the mechanism of the system. Besides, the self-made automobile heterodyne interferometer has some much more merits, for example, automobile control, a simple setup, in real-time test, reliability, and so forth. Besides, it is very stable because of its common optical path insensitive to environmental disturbances. And its feasibility is demonstrated.

\section{Introduction}

As is well known, liquid crystal displays (LCDs) have several distinct advantages, for example, light weight, thin and less volume over the conventional displays. In recent years, it has grown rapidly and been applied in many products, such as pads, smart mobile phone screen, notebook panel, PC screen, and LCD TV. If large LCDs are used for monitor and TV applications, they must be with the performance of wideviewing angle. Because of the optically compensated twisted nematic liquid crystal with the characteristics of fast response and wide viewing angle $[1,2]$, it has been used in large size LCDs for monitor and TV applications.

In fact, it is necessary for us to inspect the quality of $\mathrm{OCB}$ based on the importance of OCB with fast response and wide viewing angle. Many articles [3-6] that have been proposed to measure the cell parameters have been based on intensity detection by a photometric technique and developed relations between measured intensity and cell parameters recently. Tseng et al. presented a measuring technique [7] to determine the twist angle, pretilt angle, and cell gap of a TN LC cell based on the phase-sensitive heterodyne interferometry. Because of the measuring technique with buck optical components, Wang et al. proposed a new measuring method
[8] by phase-sensitive heterodyne interferometry to measure phase retardation versus rotational angle of an OCB cell and determine the cell parameters by curving fittings to theoretical calculations in 2010 .

In this paper, we propose a self-made automobile heterodyne interferometer with a closed control loop. Although the experimental scheme is similar to some previous articles [7, 8] on phase-sensitive heterodyne interferometry, the interferometer is to be designed as the vertical type with easy operation and test. It is worthy to be mentioned that the interferometer has a compact size because all the optical components are installed in the mechanism of the system. Besides, the interferometer has some much more merits, for example, automobile control, a simple setup, in real-time test, reliability, and so forth.

\section{Principles}

2.1. A Heterodyne Optical Source. In this paper, a heterodyne light [9] with a $2 \mathrm{KHz}$ beat frequency will be used. As shown in Figure 1, the optical structure of the heterodyne light is composed of a laser diode with the wavelength of $635 \mathrm{~nm}$, laser diode driver circuits, collimating lens, a polarizer 


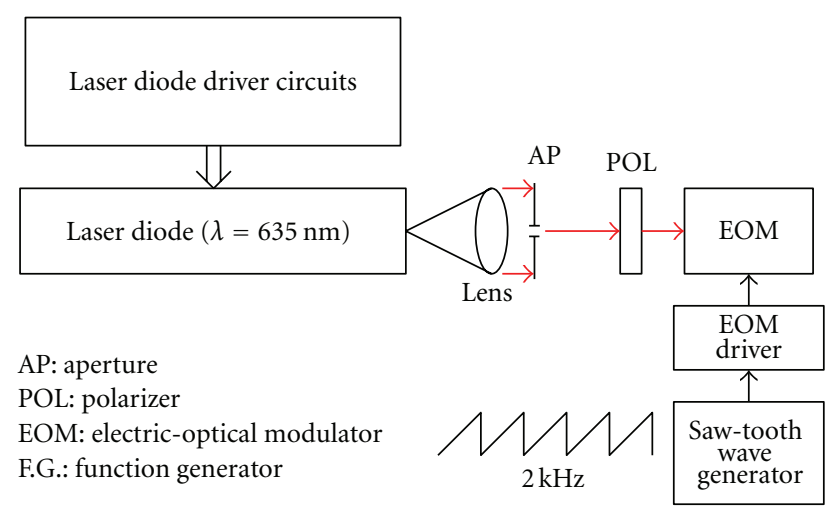

FIGURE 1: A heterodyne optical source with a $2 \mathrm{KHz}$ beat frequency.

(POL), an aperture (AP), an EOM, an EOM driver, and a saw-tooth wave generator. The EOM is modulated by driving a saw-tooth wave with the frequency of $2 \mathrm{KHz}$. For the sake of compact size, we design a saw-tooth wave generator consisting of IC TL082 and some electronic components instead of a function generator (FG).

\subsection{The Driving Circuits of Step Motor and 5-Axis Machine} Arm. At this time, let us introduce the driving circuits of step motor and 5-axis machine arm of the self-made automobile heterodyne interferometer. In the interferometer, we design a platform, that is, rotation stage, on which the tested LC can be placed. In order to rotate the platform by a step motor, the platform is designed as a round shape with 200 gears. Figure 2 shows the $3-\mathrm{D}$ structure of the platform driven by a step motor.

In the paper, a 4-phase step motor is used to drive the platform. In fact, it is necessary for us to provide a large enough driving current to push any one phase of the step motor. Figure 3 shows the driving circuits with a darling pair to drive one phase of the step motor.

In order to fetch the tested LC, a five-axis machine arm (see Figure 4(a)) manufactured by I-Chung Computer Co. Lt'd in Taiwan is used. The five-axis machine arm is with five axes including wrist, shoulder, finger, elbow, and base. Every arm can undergo either up (close) or down (open) by sending digital signals to the inputs of the arm's driving circuits composed of two photo-couplers shown in Figure 4(b). If we send 1 (or V(1)) to the photocoupler INA1 and 0 (or $\mathrm{V}(0))$ to the photocoupler INA2, respectively; thus INA1 will turn on and INA2 will turn off. At this moment, the machine arm A will be up, and vice versa.

\subsection{The Principles of Optically Compensated Twisted Nematic} Liquid Crystal. In this paper, we try to measure the parameters of a twisted nematic liquid crystal by using the selfmade automobile heterodyne interferometer. It is important for us to understand the principles of optically compensated twisted nematic liquid crystals at this time. In this paper, we want to measure the phase retardation of an optically compensated nematic liquid crystal display panel. For convenience, the liquid crystal cell is equally divided into $m$ thin

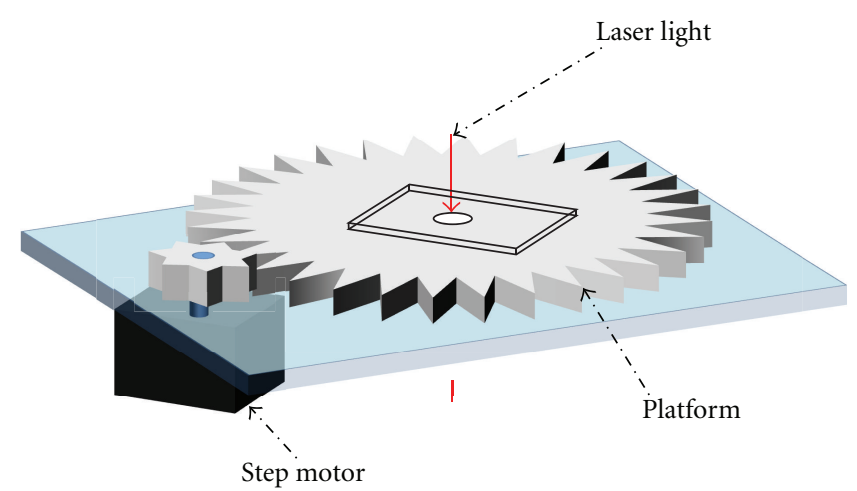

Figure 2: The 3-D structure of the platform driven by a step motor.

layers. In addition, each layer can be approximately regarded as a uniaxial wave plate if $m$ is large enough. According to the Jones matrix method, the electric field of the laser beam that passes the cell can be evaluated by multiplying all the matrixes associated with each layer in sequence as given by the following equation [8]:

$$
\begin{aligned}
{\left[\begin{array}{l}
E_{x} \\
E_{y}
\end{array}\right]=} & {\left[\begin{array}{ll}
0 & 0 \\
0 & 1
\end{array}\right] R\left(-\theta_{r}-90\right)\left[\begin{array}{cc}
e^{\mathrm{in} \cdot} \cdot 2 \pi / \lambda \cdot d / m & 0 \\
0 & e^{\mathrm{in}_{0} \cdot 2 \pi / \lambda \cdot d / m}
\end{array}\right] } \\
& \times R\left(\frac{90}{m}\right) \cdots R\left(\frac{90}{m}\right)\left[\begin{array}{cc}
e^{\mathrm{in}_{\mathrm{e}} \cdot 2 \pi / \lambda \cdot d / m} & 0 \\
0 & e^{\mathrm{in} \mathrm{n}_{0} \cdot 2 \pi / \lambda \cdot d / m}
\end{array}\right] \\
& \times R\left(\frac{90}{m}\right)\left[\begin{array}{cc}
e^{\mathrm{in} \cdot} \cdot 2 \pi / \lambda \cdot d / m & 0 \\
0 & e^{\mathrm{in} n_{0} \cdot 2 \pi / \lambda \cdot d / m}
\end{array}\right] \\
& \times R\left(\theta_{r}\right)\left[\begin{array}{cc}
\cos \frac{\Gamma}{2} \\
i \sin \frac{\Gamma}{2}
\end{array}\right], \\
& {\left[\begin{array}{c}
E_{x} \\
E_{y}
\end{array}\right] \equiv\left[\begin{array}{cc}
0 & 0 \\
p+i q & r+i s
\end{array}\right]\left[\begin{array}{c}
\cos \frac{\Gamma}{2} \\
i \sin \frac{\Gamma}{2}
\end{array}\right] }
\end{aligned}
$$

where $\Gamma$ is the phase introduced by the EOM, $\theta_{\gamma}$ is the initial azimuth angle of the rubbing direction of the cell, $R$ is the coordinate rotation matrix, $n_{o}$ and $n_{e}$ are the refraction indices of the ordinary and extraordinary waves, respectively, $d$ is the cell gap, and $\lambda$ is the wavelength of the laser diode. As the electric field of the transmitted laser beam is determined, the intensity can be express as

$$
\begin{gathered}
I \equiv|E|^{2}=E_{x}^{2}+E_{y}^{2}, \\
I \equiv C+\sqrt{A^{2}+B^{2}} \cos (\Gamma+\phi),
\end{gathered}
$$




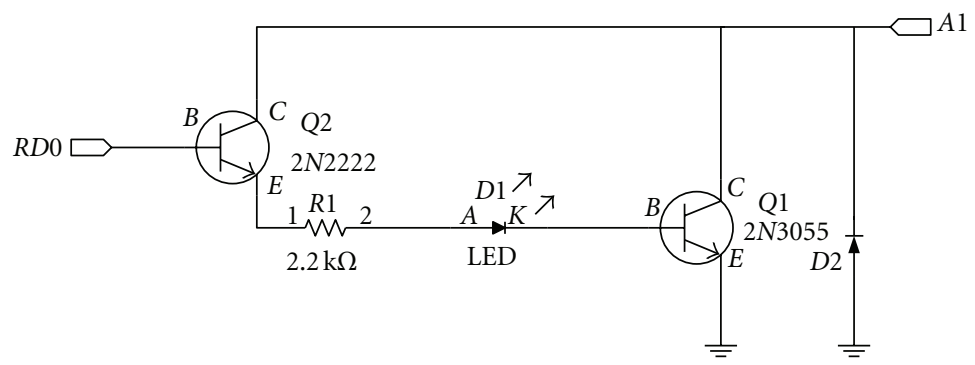

FIGURE 3: The driving circuits with a Darlington pair to drive one phase of the step motor.

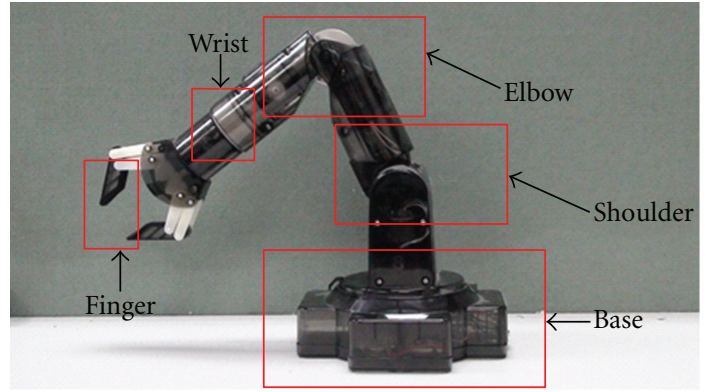

(a)

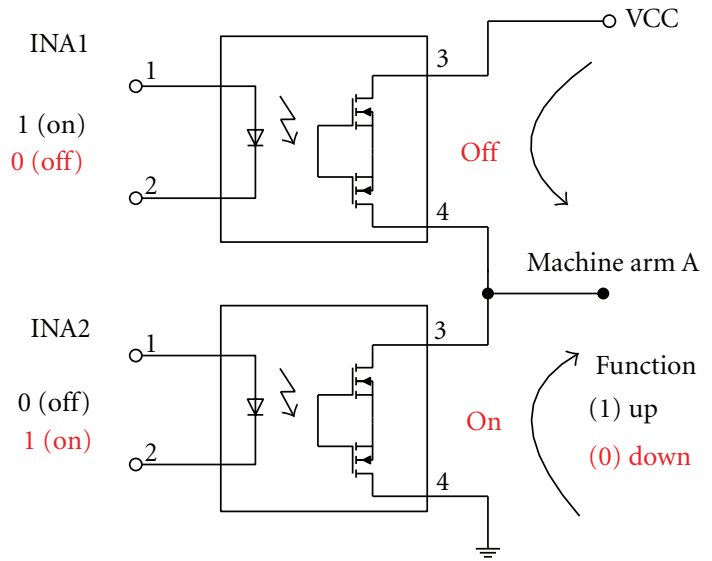

(b)

FIgure 4: (a) A five-axis machine arm manufactured by I-Chung Computer Co. Ltd. (b) The machine arm's driving circuits composed of two photo-couplers.

where

$$
\begin{gathered}
A=\frac{1}{2}\left(r^{2}+s^{2}-p^{2}-q^{2}\right), \\
B=q r-p s, \\
C=\frac{1}{2}\left(p^{2}+q^{2}+r^{2}+s^{2}\right), \\
\phi=\tan ^{-1} \frac{B}{A} ; \quad-\pi<\phi<\pi .
\end{gathered}
$$

Thus we obtain the theoretical phase retardation, $\phi$, of the optically compensated twisted nematic LC cell.

\section{Experimental Apparatus}

The experimental setup of the interferometer is shown in Figure 5. In the experimental configuration, a heterodyne light with a beat frequency $f=2 \mathrm{KHz}$ passes through the tested optically compensated twisted nematic LC and an analyzer AN and then enters a photodetector PD. The signal measured by PD is the test signal. Besides, we can obtain the reference signal from the saw-tooth wave generator. These two signals are sent to a lock-in amplifier (LIA NI USB4431 manufactured by National Instruments, USA) with the resolution $\Delta \Phi$ of $0.01^{\circ}$. Thus, we can obtain the phase retardation versus rotational angle of optically compensated twisted nematic LC cell. After some numerical computations by a computer, the cell parameters will be determined by curving fittings.

Figure 6 shows the 3-D structure of the self-made automobile heterodyne interferometer. And the photo picture of the interferometer is shown in Figure 7.

\section{Results}

In this paper, we try to measure the parameters of optically compensated twisted nematic liquid crystal (TN LC) by using the self-made automobile heterodyne interferometer. The flow chart of its program is depicted in Figure 8. As some TN LCs are prepared to be tested, the system must initialize at first. Then personal computer (PC) sends the control instruction to order the machine arm to pick up the tested LC that is placed on the unfinished region. Afterwards, the machine arm picks up the tested LC and moves it to the platform. And that the machine arm puts the tested LC on the platform, and the system begins to measure the LC. After the test has finished, the machine arm picks up the tested LC and moves it to the finished region. The system will undergo the measurement of another LC again until all of the LCs are tested completely.

In this paper, we try to measure a piece of optically compensated TN LC provided by the Research and Development Center, HannStar Display Corporation. The corresponding 


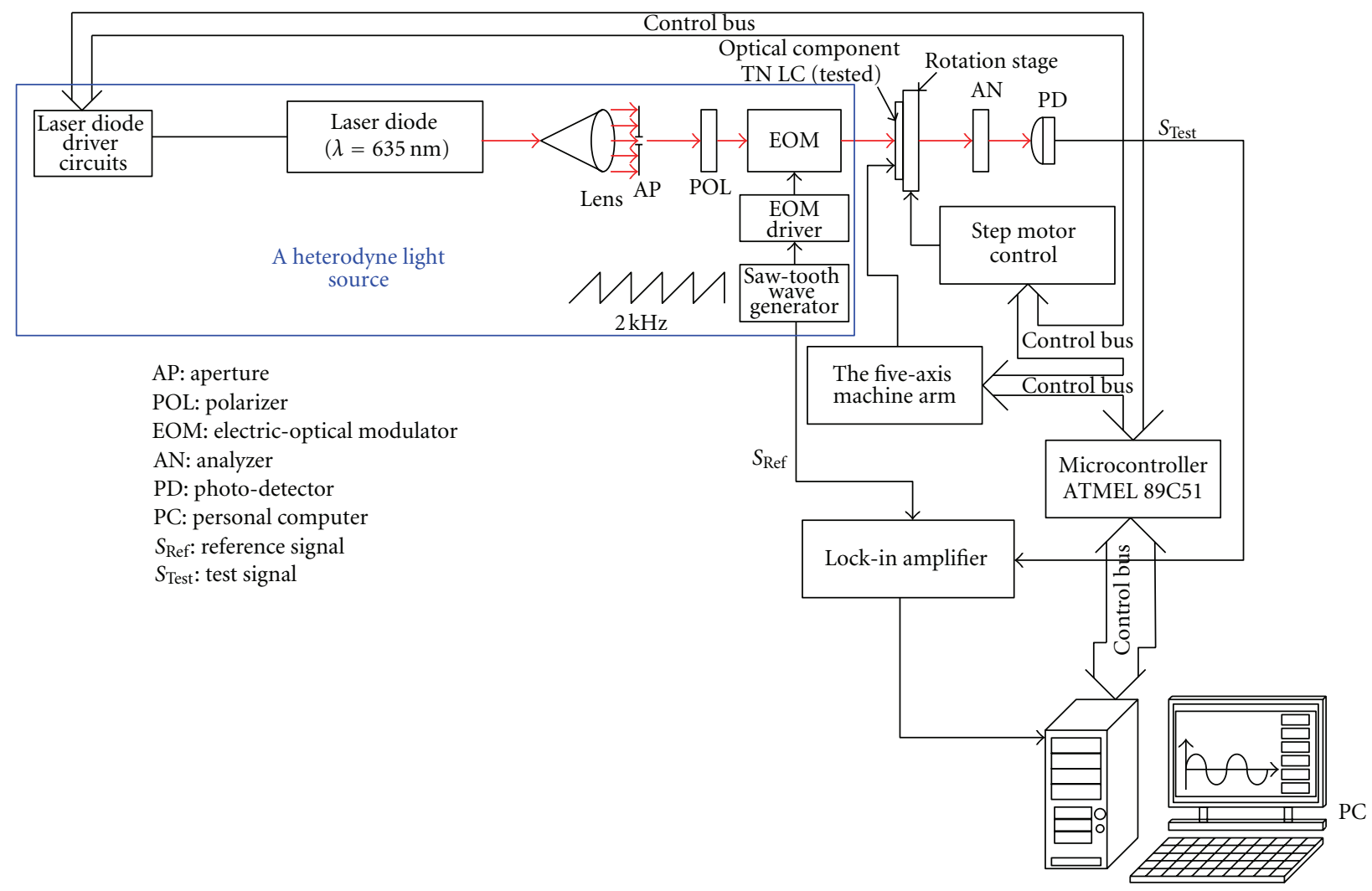

FIGURE 5: The experimental setup of the self-made automobile heterodyne interferometer.

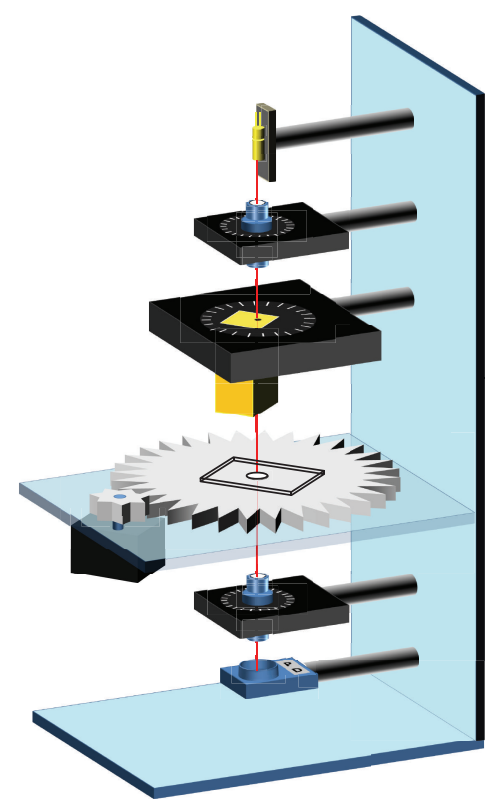

Figure 6: The 3-D structure of the self-made automobile heterodyne interferometer.

LC cell parameters such as, cell gap, pretilt angle, extraordinary refractive index, and ordinary refractive index are $3.7 \mu \mathrm{m}, 6.044$ degree, $n_{e}=1.574$, and $n_{0}=1.476$, respectively. In this paper, we apply the heterodyne interferometer

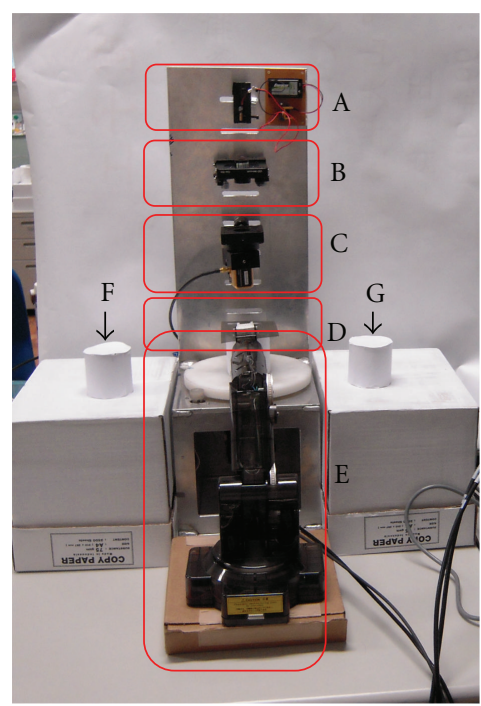

Figure 7: The photo picture of the self-made automobile heterodyne interferometer (A: laser diode driving circuits; $\mathrm{B}$ : laser diode with the wavelength of $635 \mathrm{~nm}$; C: EOM, electrooptical modulator; D: the tested LC; E: five-axis machine arm; F: the unfinished region; G: the finished region).

to measure the phase retardation versus rotational angle of an optically compensated TN LC cell.

As a matter of fact, we can measure the pretilt angle and cell gap of a TN LC cell by using the heterodyne 


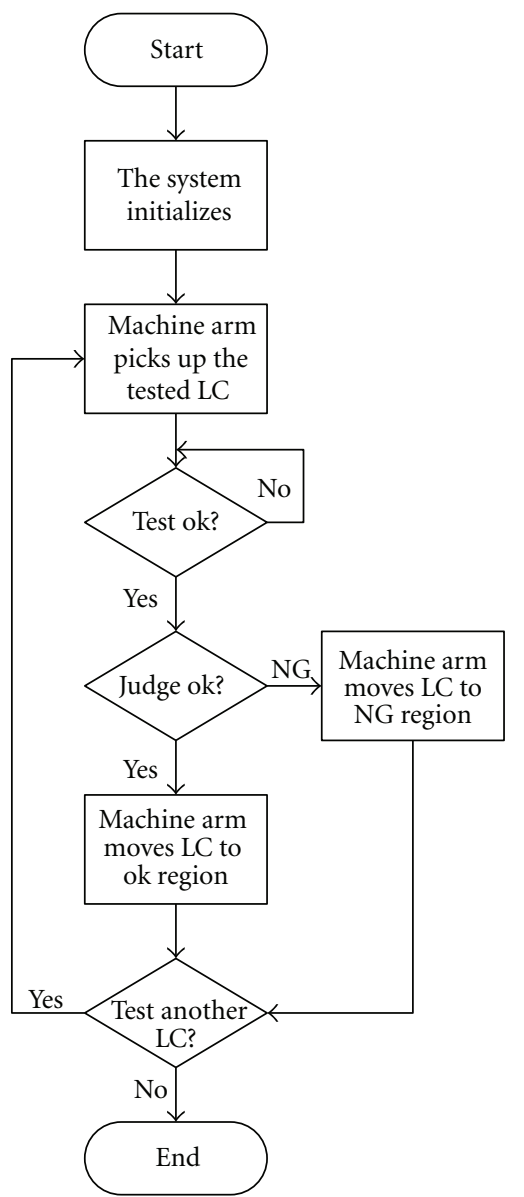

FIGURE 8: The flow chart of the control program.

interferometer. To obtain the cell parameters, we carry out a fitting procedure based on comparing the experimentally measured data with the theoretically calculated values by using iterative calculations. The experimental data and fitting curve are shown in Figure 9. From the results, the corresponding cell parameters of the optically compensated TN LC such as cell gap and pretilt angle, are determined to have $3.68 \mu \mathrm{m}$ and 6.01 degree, respectively. The data are in agreement with that of the manufacturer (cell gap equal to $3.7 \mu \mathrm{m}$ and pretilt angle equal to 6.044 degree).

\section{Discussions and Conclusion}

In the paper, a self-made automobile heterodyne interferometer with a closed control loop is proposed. However, the setup of the interferometer for measuring the phase retardation is compact, easy to operate, in real-time measurement, and simple. Besides, it is very stable because of its common optical path insensitive to environmental disturbances. In the interferometer, we design a control circuits with a closed loop by using the serial communication between the PC and MCS-51 controller. Besides, we can design an automobile precision measurement system by combining the heterodyne interferometer and some small displacement sensor [10] or small angle sensor [11]. In conclusion, we must state that

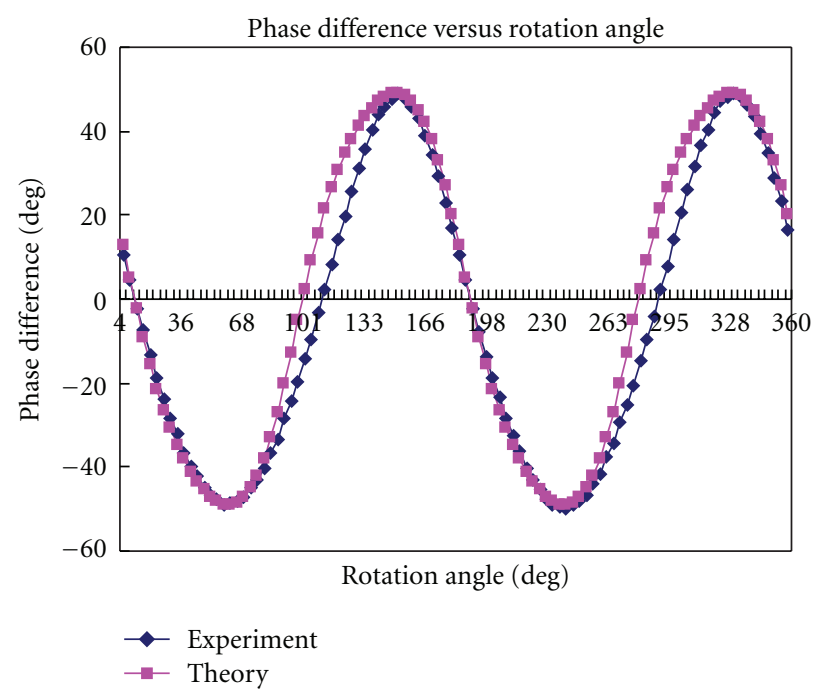

FIgURE 9: The experimental and theoretical data.

the heterodyne interferometer is original and valuable for integrated circuits (IC) industry, electrooptical industry, precision industry, and so forth. And its feasibility is demonstrated.

\section{Acknowledgments}

This research was partially supported by the National Science Council in Taiwan through Grant NSC 100-2632-E-231-001MY3 and partially supported by the Research and Development Center, HannStar Display Corporation.

\section{References}

[1] Y. Yamaguchi, T. Miyashita, and T. Uchida, in Proceedings of the Society for Information Display (SID '93), p. 277, 1993.

[2] P. J. Boss and J. A. Rahman, in Proceedings of the Society for Information Display (SID '93), p. 273, 1993.

[3] J. A. Davis, I. Moreno, and P. Tsai, "Polarization eigenstates for twisted-nematic liquid-crystal displays," Applied Optics, vol. 37, no. 5, pp. 937-945, 1998.

[4] Y. Zhou, Z. He, and S. Sato, "A novel method for determining the cell thickness and twist angle of a twisted nematic cell by stokes parameter measurement," Japanese Journal of Applied Physics, vol. 36, pp. 2760-2764, 1997.

[5] H. Kim and Y. H. Lee, "Unique measurement of the parameters of a twisted-nematic liquid-crystal display," Applied Optics, vol. 44, no. 9, pp. 1642-1649, 2005.

[6] J. S. Gwag, S. H. Lee, K. H. Park et al., "Simple method for measuring the high pretilt angle of nematic liquid crystals," Journal of Applied Physics, vol. 93, no. 8, pp. 4936-4938, 2003.

[7] H.-C. Tseng, R. B. Li, W.-C. Chen, K. H. Yang, and S.-F. Wang, "Determinations of twist angle, pretilt angle, and cell gap of a TN LC cell by phase-sensitive heterodyne interferometry," in Proceedings of the Society for Information Display (SID '08), Los Angeles Convention Center, Los Angeles, Calif, USA, May 2008.

[8] S.-F. Wang, W. Lai, J.-S. Chiu, R. H. Yeh, H.-C. Tseng, and W.C. Chen, "Method for measuring the twist angle of an optically compensation bend by using the heterodyne interferometry," 
in Proceedings of the IEEE International Instrumentation and Measurement Technology Conference (I2MTC '10), pp. 296299, 2010.

[9] M. H. Chiu, S.-F. Wang, and R. S. Chang, "Instrument for measuring small angles by use of multiple total internal reflections in heterodyne interferometry," Applied Optics, vol. 43, no. 29, pp. 5438-5442, 2004.

[10] S.-F. Wang, "A small-displacement sensor using total internal reflection theory and surface plasmon resonance technology for heterodyne interferomery," Sensors, vol. 9, pp. 2498-2510, 2009.

[11] S.-F. Wang, M. H. Chiu, C. W. Lai, and R. S. Chang, "Highsensitivity small-angle sensor based on surface plasmon resonance technology and heterodyne interferometry," Applied Optics, vol. 45, no. 26, pp. 6702-6707, 2006. 

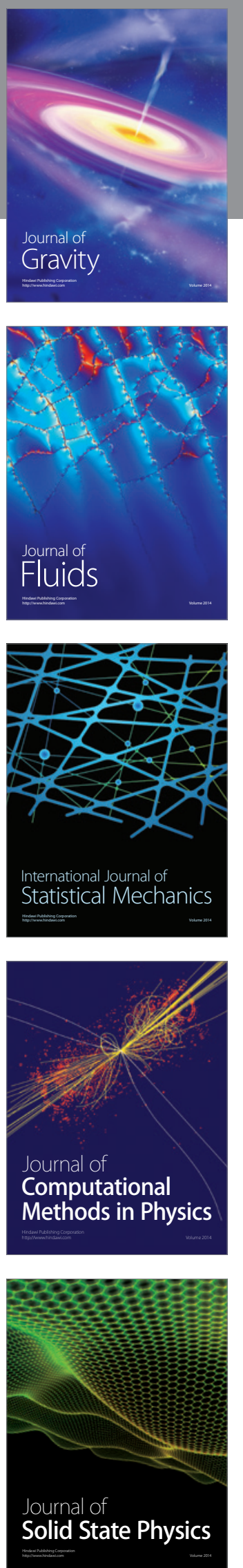

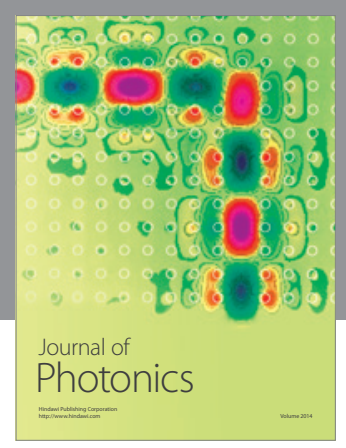

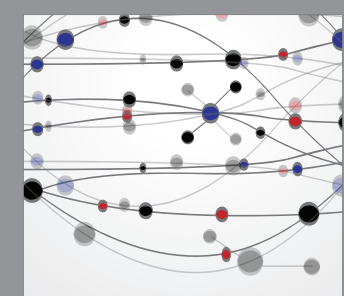

The Scientific World Journal
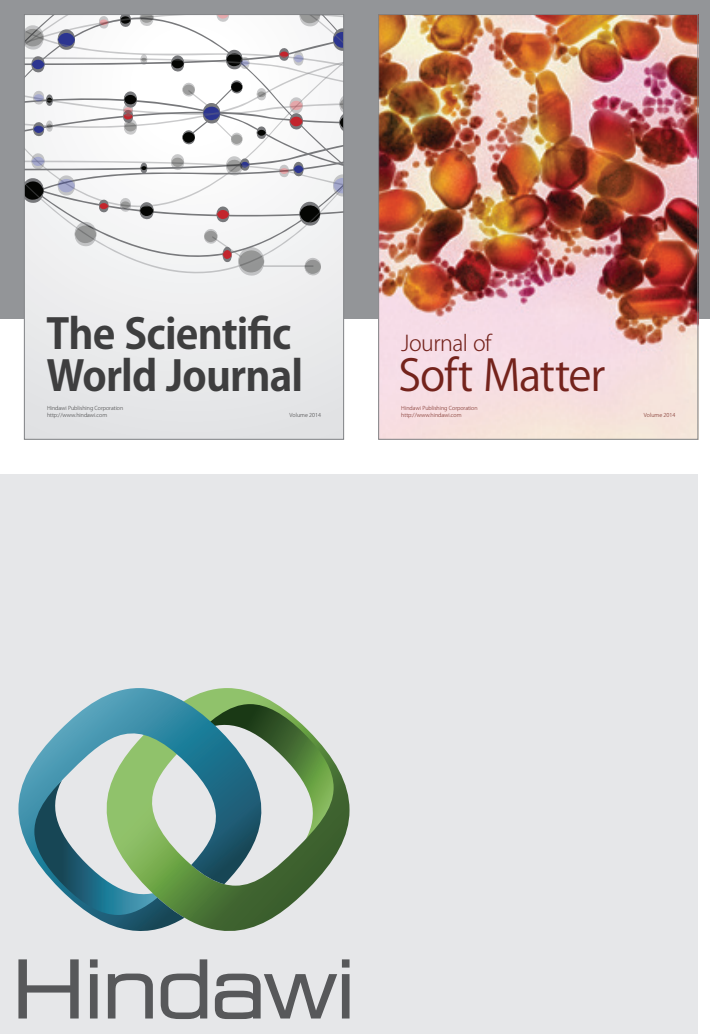

Submit your manuscripts at

http://www.hindawi.com
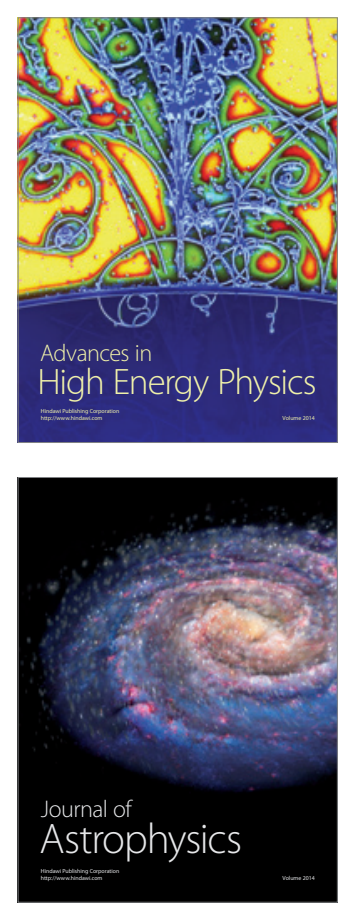
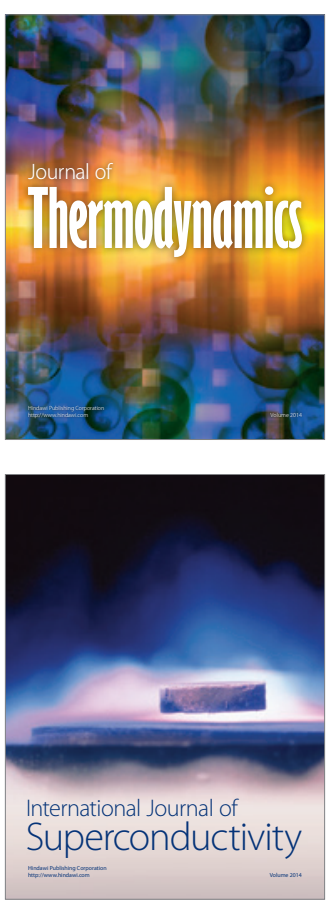
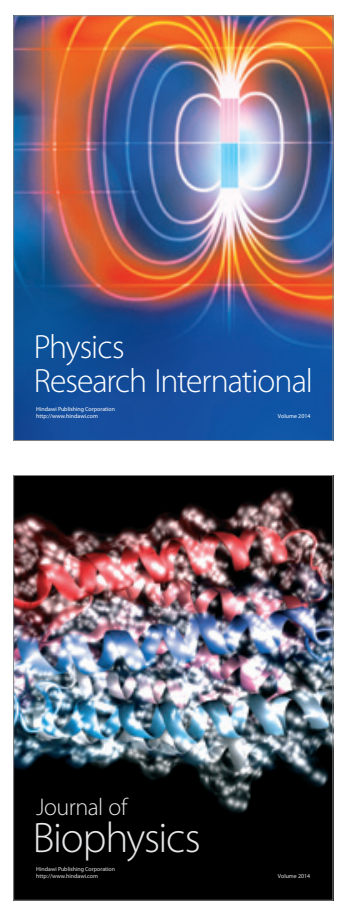
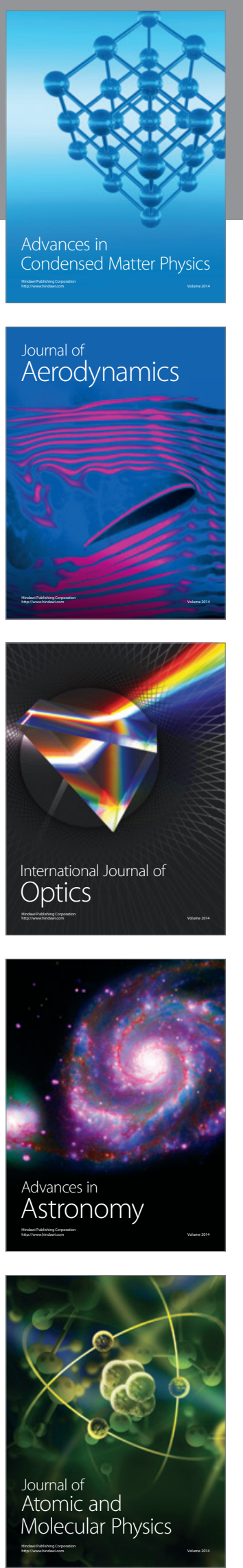